\section{P41 POSITIVE BENEFITS OF BLENDED DIET: WEIGHING IN ON GASTROINTESTINAL DYSTONIA}

${ }^{1}$ Rebecca Poole, ${ }^{1}$ Michelle Brooks, ${ }^{2}$ Jacqueline Hay, ${ }^{3}$ Konstantinos Gerasimidis, ${ }^{2}$ Sarah Bremner, ${ }^{2}$ Tracey Cardigan, ${ }^{3}$ Hazel Duncan, ${ }^{4}$ Gillian Smith, ${ }^{2}$ Elaine Buchanan, ${ }^{5}$ Simon Fraser, ${ }^{6}$ James Andrews, ${ }^{6}$ Timothy Bradnock, ${ }^{6}$ Rania Kronfli, ${ }^{6}$ Gregor Walker, ${ }^{1}$ Christina McGuckin, ${ }^{1}$ Diana Flynn, ${ }^{1}$ Andrew Barclay. ${ }^{1}$ Royal Hospital for Children, Paediatric Gastroenterology, Glasgow; ${ }^{2}$ Royal Hospital for Children, Paediatric Dietetics, Glasgow; ${ }^{3}$ University of Glasgow, School of Medicine, Dentistry and Nursing, Glasgow; University Hospital Crosshouse, Paediatric Dietetics, Kilmarnock; ${ }^{4}$ University Hospital of Wishaw, Paediatric Dietetics, Wishaw; ${ }^{5}$ Royal Hospital for Children, Paediatric Pharmacy, Glasgow; ${ }^{6}$ Royal Hospital for Children, Paediatric Surgery, Glasgow

\subsection{6/flgastro-2021-bspghan.50}

Introduction Children with severe neurodisability and tone disorders can present with debilitating symptoms and declining growth. Gastrointestinal dystonia (GID) describes the clinical manifestations of: pain behaviour; hypertonicity; retching; vomiting; vagal phenomena; abdominal distension and straining attributable to the GI tract in the context of severe neurodisability. ${ }^{1}$ Blended diet (BD) has an emerging role in the nutritional management of patients with neurodisability, however we are unaware of data pertaining to BD specifically in patients with GID.

Aim A tertiary centre review of outcomes of patients with GID receiving $\mathrm{BD}$.

Method Patients who commenced BD between 07/2017 and $02 / 2020$ were identified from prospectively gathered complex enteral nutrition (CEN) and specialist dietetic databases. BD was initiated with specialist paediatric dietetic support within the CEN clinic or in other clinics. Data gathered included: demographics; primary diagnosis; enteral feeding plan; fundoplication; weight standard deviation $\mathrm{z}$ score at $0,6,12$ and 18 months prior to and from commencing $\mathrm{BD}$; medications at 0 and 6, 12, 18 months; reasons if $\mathrm{BD}$ discontinued; parenteral nutrition (PN) requirement.

Results 29 children met criteria for GID and commenced BD. ${ }^{1}$ 18 were male. 14 had a fundoplication. Feeding method prior

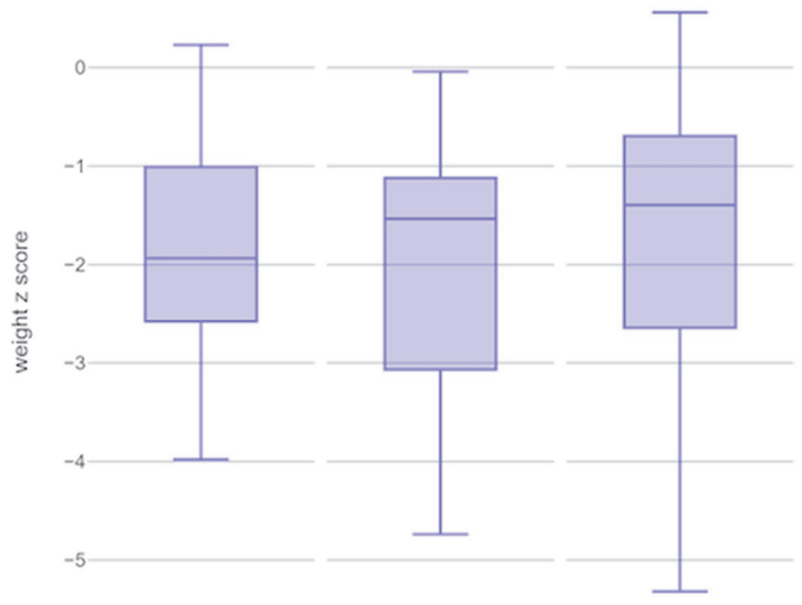

0 months $\quad 12$ months $\quad 18$ months

Abstract P41 Figure 1 Weight z scores of children with GID receiving $B D$

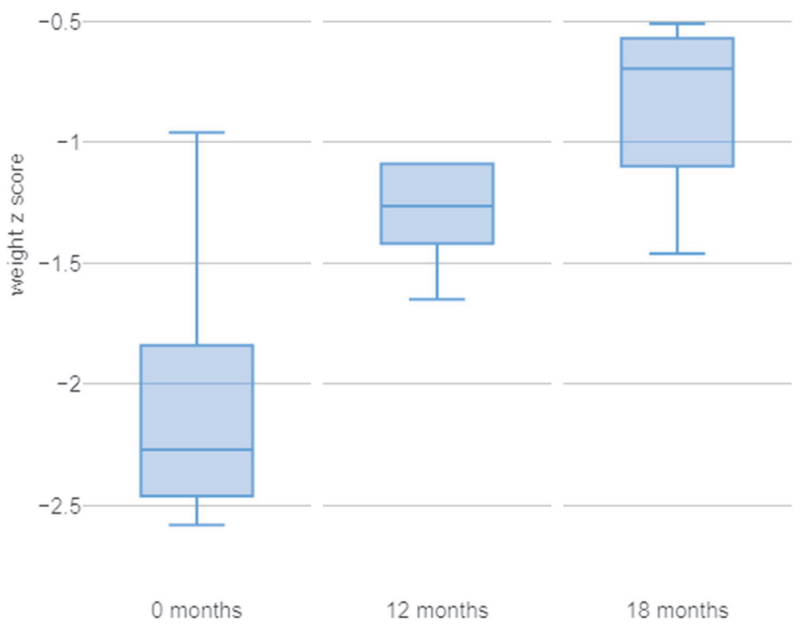

Abstract P41 Figure 2 Weight z scores of subset with GID receiving $\mathrm{BD}$

to $\mathrm{BD}$ : gastric bolus 17; gastric continuous 8; jejunal 4. Mean age $\mathrm{BD}$ commenced was 7 years. Follow up ranged 8 to 40 months. 25 patients continue $\mathrm{BD}$ to date. 4 discontinued $\mathrm{BD}$ within 1-2 months citing increased GI symptoms or device blockage.

Of the 25 who continue $\mathrm{BD}$, median weight $\mathrm{z}$ scores declined from -1.77 at 12 months prior to $\mathrm{BD},-1.86$ at 6 months prior, -1.94 at 0 months, then rose to -1.54 at 12 months, and -1.40 at 18 months. Mean weight $\mathrm{z}$ scores were maintained at -1.97 (CI -0.85 to -3.09 ) at 0 months, -1.97 (CI -0.27 to -3.02 ) at 12 months and -1.79 (CI -0.34 to $3.25)$ at 18 months [figure 1]. This trend was more significant in a subset of 6 patients, $\mathrm{z}$ score -2.06 (CI -1.46 to -2.67 ) at 0 months, -1.13 (CI -0.59 to -1.67$)$ at 12 months $(\mathrm{p}=$ 0.018 ) and -0.84 (CI -0.46 to -1.21 ) at 18 months ( $\mathrm{p}=$ 0.003 ) [figure 2].

9 of the 25 were able to discontinue one GI medication, 4 discontinued two GI medications. Tone medications: 3 reduced; 20 unchanged; 2 increased. No patients received PN during the study.

Conclusion GID represents the severest end point of gastrointestinal symptoms in neurodisability, with progressive decline often a feature. Our data show children with GID receiving $\mathrm{BD}$ continue to track their weight trajectory, whilst some experience significantly improved growth. No patients required trial on PN during the study. The authors advocate for BD's role in minimizing the need for invasive treatments in GID whilst addressing symptoms and maintaining nutritional status ${ }^{2}$ and growth superiorly to formula feeds alone. Improvements in symptomatology and quality of life will be better described by prospective survey of patients commencing BD for GID. The authors are currently gathering this data.

\section{REFERENCES}

1. McConnell N, et al. JPGN;66(supp2):1002;2018

2. Hay J, et al. (presented BSPGHAN 2020) 